\title{
Quartic Supercyclides for Geometric Design
}

\author{
Michael J. Pratt \\ Rensselaer Polytechnic Institute, USA ${ }^{1}$
}

\begin{abstract}
Most commercial CAD systems use parametric representations (e.g., NURBS) for defining free-form shapes. This leads to significant problems in certain types of geometric computation, and also in the exchange of CAD models between different systems. The paper suggests that there are good reasons for developing alternative approaches to the representation of free-form shapes in $\mathrm{CAD}$, based on the use of algebraic surfaces. Work in this direction is currently at an early stage, but some significant research directions are being identified. The paper surveys the current state of the art, and indicates some fruitful areas for future work.
\end{abstract}

Key words: Algebraic surfaces, Cyclides, Supercyclides, Blending

\section{INTRODUCTION}

All major commercial computer aided design (CAD) systems provide capabilities for defining what is known as free-form surface geometry, which is needed for the modelling of such artefacts as shoes, ships, cars, aircraft and plastic supermarket bottles. Such systems all implement either nonuniform rational B-spline (NURBS) or Bézier representations (Piegl 1995, Farin 1993). These parametric forms have the primary virtue of being defined in terms of control points that are related to the surface itself in an intuitively comprehensible way. Thus a surface designer can, in principle,

1 Currently on assignment at US National Institute of Standards and Technology. This paper is a revised and extended version of a paper presented at the WSCG99 conference on computer graphics held in Plzen, Czech Republic, in February 1999. 
manipulate the surface by moving its individual control points. However, not all CAD systems actually provide their users with this type of control. Another possible way of making local modifications to rationally represented surfaces is by adjustment of the values of the weights associated with individual control points, though no commercial CAD system known to the author allows this in practice.

The use of parametric representations of geometry leads to certain problems:

- The computation of intersection curves is complex and error-prone.

- NURBS, in particular, can sometimes give non-intuitive results from standard constructional procedures. These include the propagation of sharp crease lines into surface interiors when smooth boundaries are interpolated.

- A given surface may be parameterized in infinitely many ways, and automatically generated parameterizations may be bad for application purposes (e.g., in manufacturing by numerically controlled machining).

These difficulties, and other related characteristics of parametric surface formulations to be discussed later, motivate the study of implicit surface representations for use in CAD and computer graphics. There has recently been a rapid growth in this topic in the computer graphics community. For example, Bloomenthal (1997) has edited a book on applications of implicit surfaces in computer graphics and animation. However, there has so far been less work on their application in $\mathrm{CAD}$, where it appears that algebraic surfaces rather than general implicit surfaces provide the best way forward.

The degrees of the parametric surfaces used in current CAD systems routinely range from $3 \times 3$ to $15 \times 15$, with one system going as high as $20 \times$ 20. If we consider the Bézier representation, an $n \times n$ surface patch has $4(n$ $+1)^{2}-1$ degrees of geometric freedom, since there is a 4-vector associated with each control point but one component of one of those vectors may be chosen arbitrarily. Six of the remaining degrees of freedom are concerned with positioning and orienting the surface patch in space. Taking the view that pure shape is what we are concerned with, we can subtract these as well for purposes of comparison. Then the $3 \times 3,15 \times 15$ and $20 \times 20$ patches have respectively 57, 1017 and 1757 degrees of freedom.

Thus, if we consider the spectrum of surfaces commonly used in CAD, we find the following:

- plane - algebraic degree 1; 0 shape variables

- right circular cylinder - algebraic degree 2; 1 shape variable (radius)

- sphere - algebraic degree 2; 1 shape variable (radius) 
- right circular cone - algebraic degree $2 ; 1$ shape variable (apex angle)

- torus - algebraic degree 4; 2 shape variables (major and minor radii)

- Dupin cyclide - algebraic degree 4; 3 shape variables (two generalized radii, asymmetry variable)

- rational parametric bicubic surface - algebraic degree $\leq 18 ; 57$ shape variables

- ...

- rational parametric $20 \times 20$ surface - algebraic degree $\leq 800 ; 1757$ shape variables.

Clearly there is a rather sudden jump in algebraic degree and geometric freedom as we leave the most complex of the 'simple' surfaces and progress to the simplest of the parametric surfaces. This fact has motivated the author to examine possible extensions of the range of 'simple' surfaces, in an attempt to find surfaces that are of algebraically low degree and are controlled by a relatively small number of shape variables that can preferably be intuitively understood by a CAD system operator. So far the search has had limited success, but it is hoped that this paper will show that there are signs of progress.

To gain further insight, we may consider how the geometric properties of parametric surfaces vary with their parametric degree. In fact not much is known about this, but it is known that every rational parametric surface can also in principle be expressed as an algebraic surface (though the converse is not true - not all algebraic surfaces possess a rational parameterization). A parametric surface representation based on four-sided patches, with degree $n$ in its parameters $u$ and $v$, has a corresponding algebraic representation of degree $\leq 2 n^{2}$ (Sederberg 1984) ${ }^{2}$. Geometrically, this means that a given straight line may intersect the surface in as many as $2 n^{2}$ points, so that the algebraic degree gives some measure of the possible undulation of the surface. The maximum algebraic degrees of surfaces with parametric degree $3 \times 3,15 \times 15$ and $20 \times 20$ are respectively 18,450 and 800 (two of these figures were shown in the comparison list above). The equation of a general algebraic surface of degree $N$ contains $1 / 6(N+1)(N+2)(N+3)$ coefficients, of which one may be chosen arbitrarily, the rest each representing one degree of geometric freedom. For the parametric surface of degree $3 \times 3$ the number of coefficients is 1330 . For the higher-degree surfaces the number of coefficients becomes phenomenal by the standard of current computations with polynomial functions, which gives some indication of the scale of the

2 Correspondingly, for a parametric surface based on three-sided patches and having degree $n$ in each of its three parameters, the corresponding algebraic degree is $n^{2}$. 
practical problem of actually computing the algebraic form of a given parametric surface.

However, it was noted above that (i) the rational parametric surfaces correspond to a subset of the algebraic surfaces, and that (ii) the degree of the algebraic representation of a parametric surface may be less than the maximum given in the preceding paragraph. It is therefore possible that the oscillatory behaviour of a given parametric surface may be much more benign than its maximum possible algebraic degree would indicate, but this cannot be assumed in the absence of theoretical justification. It appears that little work has been done on the determination of geometric properties of surfaces in terms of their parametric representations. A paper by Warren (1990) gives some indication of how such an investigation might proceed, but for the moment it seems best to urge caution in the use of surfaces with very high parametric degree. In practice, the use of such surfaces is usually restricted to tightly constrained situations, where many degrees of freedom are necessary to meet the constraints. Even so, indications are that these surfaces provide many more degrees of freedom than can possibly be required.

It is also instructive to consider the geometry of intersection curves. Two surfaces of algebraic degree $N$ intersect in a curve of degree $N^{2}-$ this is a measure of oscillatory tendency, since it represents the number of times the curve may intersect with a plane. Thus, two surfaces having rational parametric degree $n \times n$ intersect in a curve of maximum algebraic degree $4 n^{4}$. For two bicubic parametric surfaces this degree is $\leq 324$; for two CATIA surfaces of parametric degree $15 \times 15$ it may exceed $2 \times 10^{5}$. Again, these upper bounds are possibly very pessimistic, but they do provide some theoretical grounds for the practical difficulties met with in computing intersection curves between parametric surfaces.

It appears, then, that current CAD systems are using inappropriate types of geometry. The very high algebraic degree of such surfaces makes it very difficult to devise geometric algorithms that are not only robust but also fast and accurate. For example, small oscillations in a surface may give rise to small closed loops in an intersection curve that are hard to detect computationally and may be missed. The structural integrity of boundary representation (Brep) solid models, in particular, can suffer because of this and similar difficulties. Errors in intersection computations can cause the geometry of the model to be incompatible with its associated topological (connectivity) information. Such problems have been highlighted by experience with the CAD model exchange standard ISO 10303 (ISO 1994, Owen 1997), informally known as STEP, (STandard for the Exchange of Product model data). STEP exchanges can be incomplete or can fail 
altogether because of geometry/topology discrepancies in transmitted models.

In view of the problems described above, it is natural to suggest that it may be possible to achieve the necessary flexibility for free-form design, while also achieving better modeling robustness, through the use of algebraic surfaces of comparatively low degree. The major difficulty, as will be seen, lies in finding a suitable means for controlling the shape of algebraically defined surfaces.

At present there is little motivation to turn to the use of non-rational parametric surfaces or non-algebraic implicit surfaces for CAD applications. The reason for this is that rational functions ${ }^{3}$ are the most general functions that can be evaluated in principle using the four basic computational operations of addition, subtraction, multiplication and division. The use of any other functions would introduce inevitable additional errors of approximation in the evaluation of surface geometry, jeopardizing the representational accuracy that is crucially important in many engineering applications of CAD. However, as mentioned earlier, non-algebraic implicit surface representations are becoming popular for use in computer graphics and animation (Bloomenthal 1997).

It must be emphasized at the outset that the work reported here is very preliminary. Little has so far been done on free-form shape modelling using the class of surfaces proposed in the paper, and the geometric continuity that can be expected from their use is restricted to $G^{1}$. Nevertheless, it is hoped that similar algebraic approaches can be identified for modelling with $G^{2}$ continuity, possibly using surfaces of higher degree.

\section{CHANGE OF REPRESENTATION}

Some facts regarding the relation between algebraic and parametric surfaces will be briefly related here. First, any parametric surface with a rational parameterization corresponds to an algebraic surface, as mentioned above. The process of deriving the algebraic from the parametric form is called implicitization. Several methods for this have been investigated, mostly based on the use of resultants, but they are only practical for parametric surfaces of low degree, because otherwise the resulting algebraic surfaces will have immensely high degree (Sederberg, Anderson and Goldman 1984, 1985).

On the other hand, not all algebraic surfaces possess a rational parameterization, though they may have parameterizations expressible in

\footnotetext{
${ }^{3}$ Here and in what follows, rational functions will be taken to include polynomials.
} 
terms of fractional powers or transcendental functions. The inverse process to implicitization, called parameterization, is thus in general not possible if a rational parametric form is desired. In practice there is some virtue in working with surfaces that have both an algebraic and a parametric form, since the simplest approach to the calculation of intersections between two surfaces becomes possible when one is expressed implicitly and the other parametrically (Pratt 1986). Intersection calculations are frequently required during the generation of a CAD model, and it is important that they can be performed quickly, robustly and accurately. This requires that algorithms are efficient in handling two types of complexity:

- global complexity - the existence in an intersection curve of numerous disjoint branches, some of which can be small closed loops, difficult to detect.

- local complexity - the existence in an intersection curve of singularities such as cusps, self-intersections, self-tangencies, etc. These can all confuse the kind of 'marching' algorithms usually used in practice for tracing intersections.

It should be noted that the higher the algebraic degree of the intersecting surfaces, the more likely it is that these problems will occur.

\section{ALGEBRAIC SURFACES IN CAD}

\subsection{The current situation}

All CAD systems implement the following 'simple' surfaces: the plane, right circular cylinder, right circular cone, sphere and torus. The plane has a linear equation, and all the others except for the torus are second-degree (quadric) surfaces. The torus is a fourth-degree (quartic) surface. A few systems provide more general quadrics (e.g., elliptic cones) and more general quartics, in particular the Dupin cyclides, of which more later.

Although these are all algebraic surfaces they are not usually represented in CAD systems in their algebraic forms, though these may be generated if required for certain types of calculation. Typically such a surface is represented either in a NURBS form or in terms of what is called its geometric representation, in terms of a small number of shape-defining entities. Thus a plane is often defined in terms of a point and a normal direction, a cylinder in terms of a centre-line and a radius, a cone in terms of 
an apex point, a centre-line and an angle, and a sphere in terms of a centrepoint and a radius. The torus is characterized by a centre-point, a centreline, and major and minor radii.

One of the problems of working with more general algebraic surfaces is that it is difficult to identify such convenient geometric 'handles' for their manipulation. This is where the NURBS and Bézier parametric surface representations do have a distinct advantage; they allow the design and modification of surface geometry in terms of 'control points' whose relation to the surface is fairly easy for the designer to comprehend. We will return to this matter of geometric control in a later section.

\subsection{Choice of surface degree}

If attention is focused on free-form design, overall surfaces will often be modelled in terms of patches of simpler surfaces joining with some prescribed level of geometric continuity. For some applications simple $G^{1}$ (tangent plane) continuity of adjoining patches will be adequate. For others, e.g. car body design, $G^{2}$ (surface curvature) continuity may be demanded. Such requirements will affect the choice of degree of the algebraic surfaces to be used. Most research to date has concentrated on the achievement of $G^{1}$ continuity. Even in this case there is so far no unanimity of opinion on the choice of degree giving the optimal compromise between geometric flexibility and intuitive designer control.

There is fairly general agreement that the quadric surfaces do not allow sufficient geometric freedom for freeform design, though there has recently been an investigation into their use for computer graphics modeling (Froumentin 1997). Cubic algebraic surfaces have been studied by Sederberg (1990). He developed a means of controlling them for design, though not in a manner that would be intuitive for a non-mathematical designer. Sederberg also noted an inherent problem of algebraic surfaces in general; the topology of the surface can change significantly and unexpectedly as coefficients of the defining equation are varied.

Whereas the algebraic cubic is unbounded, the parametric patch is a bounded surface region, but that is because the patch is a mapping of a finite parameter domain into Euclidean space $\mathbf{E}^{3}$. The same representation gives an unbounded surface if its extension to an infinite parameter domain is considered, though the behaviour of the surface outside its original domain of definition is usually of no interest to the CAD designer. By contrast, the algebraic surface is usually regarded as an unbounded entity, and one of the problems of using it in CAD is finding suitable means for defining patch boundaries on it. Thus the parametric formulation includes patch boundaries as an integral part of geometry definition, whereas the pure algebraic 
approach appears to demand additional patch boundary information to supplement the surface specification itself. This shows the advantages of working with algebraic surfaces that have a natural associated parameterization, such as those discussed below.

\subsection{Quartic surfaces}

Since the cubic surfaces appear to show little promise for CAD, attention has turned to the quartics. The general quartic algebraic surface has 34 degrees of freedom, and it is consequently difficult to provide intuitive means for its geometric control. The study of the quartics for use in CAD has therefore been restricted to special cases, some of which show promise for free-form design. Sederberg and Anderson (1985) have examined the Steiner surfaces, which provide only limited geometric flexibility and are not easy to control. They have the added disadvantage that they naturally give rise to triangular patches, whereas quadrilateral patches are preferred in CAD. The Dupin cyclides (Dutta 1993) are a class of quartic surfaces that are easier to handle from the designer's point of view, having a 'geometric' representation in terms of three shape-defining variables. There are also cubic forms (see Figure 1). They have been found useful for the construction of blend surfaces in solid modeling (Pratt 1990, 1995), and they give rise to quadrilateral surface patches, appropriate for CAD use, though Martin et al. (1986) found that they give insufficient freedom for fully free-form design.

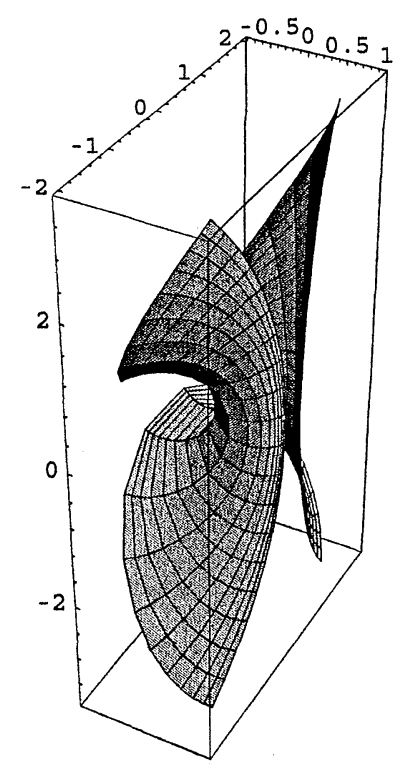

Figure 1: Part of a cubic Dupin cyclide 
The Dupin cyclides are of particular interest in that they provide an incremental increase in the range of 'simple' surfaces implemented in CAD systems. They are generalizations of the torus, having the same (quartic) algebraic degree and just one additional degree of geometric freedom.

More recently interest has arisen in supercyclides, generalizations of the Dupin cyclides (Degen 1994, Pratt 1996, 1997). These include projective transformations of the quartic Dupin cyclides and their degenerate cubic cases, and therefore permit greater freedom for shape modeling.

The supercyclides (see Figure 2) can be parameterized by a conjugate net of conic curves, and they have the additional property that their tangent planes around any such curve envelope a quadric cone. In the special case of the Dupin cyclides the conic curves are circles and the tangent cones right circular cones. The existence of these cones makes it easy to join supercyclide patches with tangent continuity into larger surfaces defined in a piecewise manner - it is only necessary to match two supercyclides to the same cone along a shared conic curve. The blending of quadric surfaces in solid modeling can be based on the same principle (Allen 1996, Degen 1994).

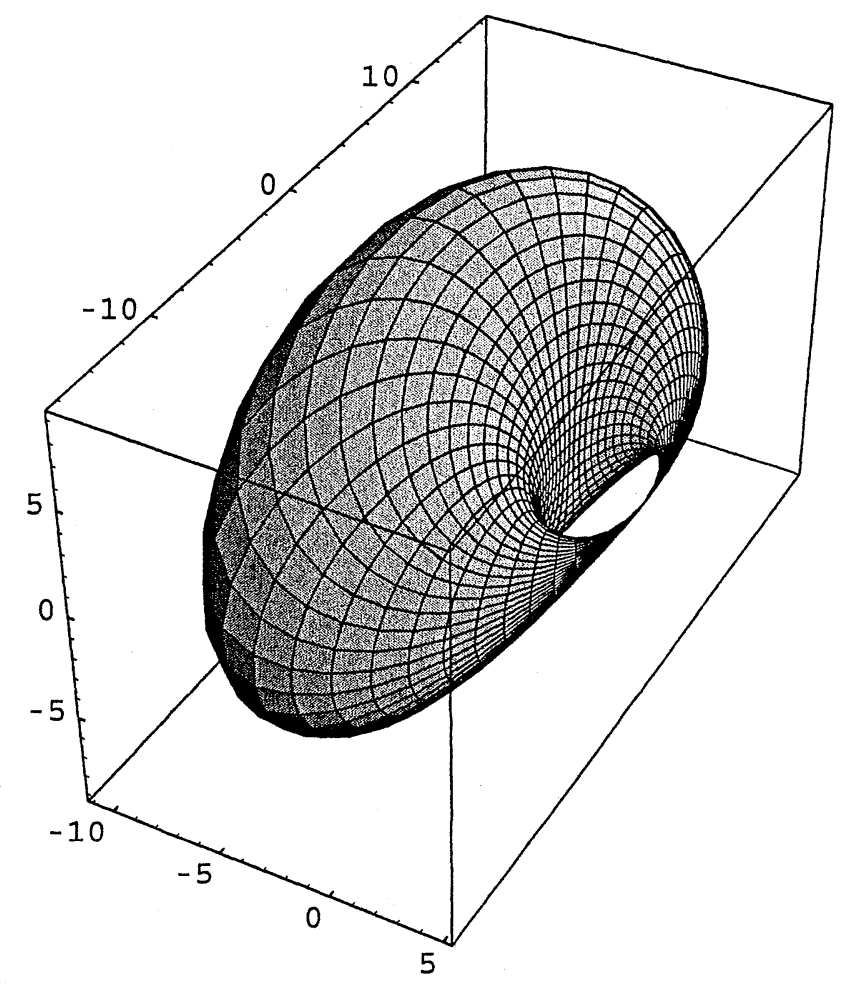

Figure 2: Example of a supercyclide ("ellipside") 
Supercyclides, unlike Dupin cyclides, may provide sufficient geometric freedom for fully free-form surface design. The fact that they generate quadrilateral patches with rational biquadratic parameterizations (Pratt 1996, 1997) also makes their use compatible with existing standards for CAD data transfer such as STEP, referred to earlier ${ }^{4}$. This standard does not provide a means for transferring triangular patch data, since there are no current CAD systems that use such patches to define free-form surfaces. Some systems do provide non-standard $n$-sided patches, with $n \neq 4$, for occasional use in specialized applications such as blending sharp vertices of 3D models (Malraison 1998), but these have to be approximated in terms of more standard representations for purposes of data exchange between CAD systems.

A further advantage for the CAD use of Dupin cyclides and supercyclides lies in the existence of exact solutions to the inverse parameterization problem, i.e., the determination of parameter values for a given cartesian point on a surface (Pratt 1997). This type of calculation is a frequent requirement in geometric computations for CAD. Supercyclides have the further property that patches bounded by isoparametric curves on them have coplanar corners. This makes it almost trivial to generate approximate representations to any desired accuracy in terms of planar quadrilateral facets. Such a characteristic is useful for many purposes, including the efficient generation of graphical renderings. It also has potential as the basis of a subdivision method for computing intersections between supercyclide surfaces.

An additional class of quartic surfaces that may prove useful in CAD is another family of generalized cyclides extensively studied in the 19th century by Darboux (1896). These have recently arisen in some work by Paluszny and Boehm (1998). They are especially interesting because, although their algebraic degree is only four, they appear to permit $G^{2}$ blending in certain circumstances where the Dupin cyclides would only provide $G^{1}$. Clearly, great scope exists for further investigation of the Darboux cyclides for shape design.

It may be concluded that certain classes of quartic algebraic surfaces show good potential for application in CAD. Most research to date has focused on the supercyclides (including the Dupin cyclides), which fit very well into the context of existing CAD systems. These may prove sufficient for the modeling of shapes in which only $G^{1}$ surface continuity is required. Surfaces with algebraic degree higher than four may be needed for the achievement of higher orders of continuity in piecewise defined surfaces in

${ }^{4}$ It is worth noting that the second edition of Part 42 of ISO 10303, 'Geometric and topological representation', includes a limited representation of the Dupin cyclide intended primarily for use in the modelling of ductwork in heating and ventilation systems. 
general. The supercyclides are discussed in more detail following this general survey of algebraic surfaces in CAD.

\subsection{Triangular patch methods}

When working with general algebraic surfaces rather than cyclides and their generalizations, it proves easier to work with triangular rather than quadrilateral patches. Several approaches have been described for the construction of piecewise surfaces from triangular algebraic patches (Bajaj 1993, Dahmen 1993, Guo 1991, Sederberg 1985). However, these are oriented more towards the generation of surfaces interpolating point and tangent data rather than design in any more usual sense. Some of these methods use patches of mixed degree, from three through five. Menon (1994) has shown how low-degree algebraic surfaces can be used in constructive solid geometry (CSG), by building volumes with curved threesided outer faces onto an initial triangulated polyhedral approximation of the desired shape. Although the main theme of this paper is concerned with quadrilateral patch methods as used in current CAD practice, the subsidiary use of triangular patches is likely to be advantageous in handling those special situations where $n$-sided regions need to be filled (Malraison 1998).

\subsection{Pure blending}

Apart from the work already cited, many workers have studied the use of algebraic surfaces purely for blending purposes (Rockwood 1997). However, the intention of this paper is to highlight the potential for research that may lead to a more general shape design capability using algebraic surfaces, with consequent improvements in accuracy and robustness resulting from the elimination of the unwarranted degrees of freedom associated with parametric geometry.

\subsection{Further details of the quartic supercyclides}

The history of the study of supercyclides is as follows. They were discovered by Degen $(1982,1986)$, who took as his starting point an article by Blutel (1890). This demonstrated the existence of surfaces bearing a family of conics, such that the tangent planes of the surface along any conic of the family envelope a quadric cone. Degen, in his two papers, used a constructive analysis to show that surfaces exist that have double possession of the Blutel property, i.e. bear two families of conics with the tangent cone property. He also derived parametric representations for them. 
In 1989 the present author was studying the CAD uses of Dupin cyclides, for which the families of conics both consist of circles and the tangent cones are right circular cones (Pratt 1990, 1995). At this time Degen introduced him to the 'double-Blutel surfaces' as generalizations of the Dupin cyclides. A subsequent study of $19^{\text {th }}$ century work by Kummer (1864) - see also Pratt (1997) - then revealed that most quartic cases of these generalized cyclides (renamed 'supercyclides' by this time) are quartic surfaces with a singular (double) conic and four additional isolated singularities.

For the special case of the quartic Dupin cyclides the double conic is the circle at infinity and there are two pairs of isolated singularities, one real and one complex. The nature of the double conic makes the calculation of the intersection between two Dupin cyclides comparatively simple, because one quartic component (the shared double circle at infinity) is always known, and it is only necessary to solve for the remaining quartic curve. Unfortunately this property is lost for the supercyclides, since in general they have different double curves.

The analysis of the quartic supercyclides from Kummer's standpoint gave rise to both implicit and parametric formulations for them, the latter agreeing with the earlier results of Degen (Pratt 1997). However, there are some special cases of the quartic supercyclides for which the double curve is a line, and cubic supercyclides also exist; these do not fit into Kummer's framework, and are the subject of continued investigation by the present author (Pratt 2001). In the meantime Degen (1998) has given an analysis of the entire family of supercyclides starting from an algebraic variety originally defined in the projective space of five dimensions. Projections of this variety from a point are intersected with a hyperplane to yield the supercyclides and explain the origin of their key properties.

On the practical side, the use of supercyclides as blending surfaces in shape modelling has been investigated by Degen himself (Degen 1994), by Allen and Dutta (1997), and by Shene (1997). To the author's knowledge, their use for the definition of general free-form surfaces has not yet been studied.

\subsection{Classes of supercyclides and their properties}

As mentioned above, there are three main classes of supercyclides. Two contain quartic surfaces, and one contains cubics. Their distinctive features are briefly stated below, following a review of the general properties of supercyclides.

The parameterization of these surfaces in terms of two families of conics, and the possession of a quadric tangent cone along any such conic have already been mentioned. The existence of the tangent cones, which of 
course are developable surfaces, implies that the two families of generating conics are mutually conjugate (Weatherburn 1927). More specifically, each family of generating conics lies on the planes of a pencil; the axes of the pencils are known as the characteristic lines of the surface.

Corresponding points on any two conics of the same family are subject to a perspective relationship, the point of projection lying on the characteristic line of the other family of conics (Degen 1994). In the limit, as the two conics become arbitrarily close, this property reduces to the tangent cone property, and the axis of the tangent cone therefore also lies on the second characteristic line.

Any supercyclide patch bounded by segments of two conics from each family has coplanar corners - this is an immediate consequence of the perspective relationship mentioned in the previous paragraph. Such a patch has a biquadratic Bézier representation in which the central control point lies at the common intersection of the tangent planes at the four patch corners (Degen 1994, Pratt 1995).

- quartic cases - Here there are two subcases,

- with a singular conic: These cases belong to the family of quartics studied by Kummer (1864). They have two pairs of singular tangent planes (tropes), tangent to the surface along an entire conic, and they possess a singular conic. Figure 3 shows a patch of such a supercyclide, including part of its singular curve.

- with a singular line: These cases are in some sense intermediate between those just described and the cubic cases. They have only one pair of tropes, which intersect in one of the characteristic lines. The singular conic is replaced by a double line lying in the surface, and this acts as the second characteristic line. There is a plane that is tangent to the surface along the double line.

- cubic cases - For these cases both characteristic lines lie in the surface. There are no tropes, and the surfaces have no singular curve. 


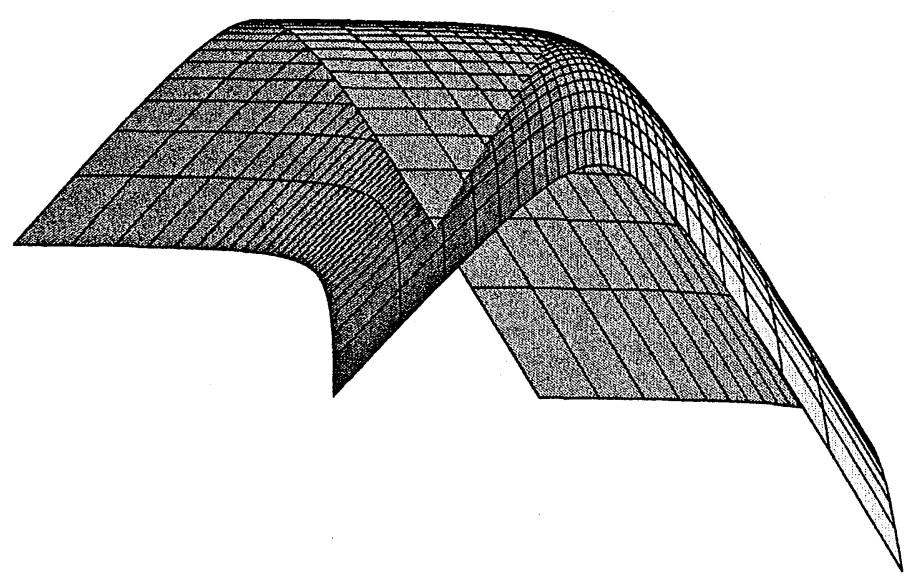

Figure 3: Quartic supercyclide patch exhibiting singular curve

All forms of supercyclide exhibit general cases having skew characteristic lines, and special cases with intersecting characteristic lines. The special cases have distinctive parametric forms (Pratt 2001).

The quartic supercyclides include projective transformations of the quartic Dupin cyclides. These cases are distinguished by the possession of an irreducible singular conic ${ }^{5}$. The cubic cases similarly include projective transformations of the cubic Dupin cyclides. In both cases consistency conditions exist that determine whether or not this is the case. For the supercyclides characterized by a singular line it is not yet clear whether there exists a coresponding 'canonical' surface whose isoparametric lines are all circles.

\subsection{Practical aspects of working with supercyclides}

The simplest way to enable a user to work with supercyclides is to take advantage of the rational biquadratic parametric representation these surfaces have, and to provide manipulation in terms of patches and control points. However, important higher-level capabilities can be provided

5 It is evident that if the conic is degenerate it cannot be obtained by projective transformation from the double curve of the Dupin cyclide, which is the circle at infinity. 
automatically, particularly in respect of joining patches with $C^{1}$ continuity, when the tangent cone property of patch boundaries can be used to derive necessary geometric properties of patches to be joined to an existing patch or patch complex. Maintenance of continuity characteristics as control points are manipulated will ensure that geometric changes do not occur at an unacceptably local level; instead, they will be smoothed out over a region of the composite surface. One way of creating a composite supercyclide surface would be to base it on two intersecting families of $G^{1}$ piecewise conic curves, and then to interpolate a supercyclide patch into each foursided mesh cell. The curves of the initial network could be constructed by generalizing a technique described some years ago by Várady (1983). He defined a double-quadratic curve segment composed of two non-rational parametric quadratic sub-segments joined with $G^{1}$ continuity at its parametric mid-point. This proves to have the same characteristics for interpolation as a parametric cubic curve segment, and a rational version of it can therefore be used to provide a suitable framework for a piecewise supercyclide surface. Each mesh cell will then be bounded by four rational double-quadratic curves, and an assembly of four supercyclide patches will be fitted into each such cell. The resulting number of individual patches is likely to be larger than when parametric surfaces of higher degree are used, but this disadvantage may be offset by improved geometric robustness. There is also the potential for development of geometric algorithms specifically tailored for use with supercyclide surfaces (for example, making use of their alternative algebraic formulation).

The supercyclides can be guaranteed to behave in predictable ways when used in CAD. It was earlier mentioned that they may have up to four isolated real singular points, but such a point can only appear in a patch interior if the patch boundaries intersect, a situation that is easy to avoid. Every supercyclide has a conic curve of self-intersection, which may be complex or at infinity, but here again it is only necessary to ensure that the boundaries of a supercyclide patch are well-behaved to exclude any real selfintersection from the patch interior. Furthermore, since the isoparametric curves of a supercyclide are conics with smooth and predictable curvature distributions, unwanted regions of high surface curvature are unlikely to arise.

\section{CONCLUSIONS}

Significant research is needed to perfect the use of algebraic surfaces for the modeling of free-form shapes in CAD and computer graphics. However, this paper has shown that some of the foundations are in place. The reward 
for solving the problems of modeling with algebraic surfaces will be more robust and efficient geometric algorithms, which should help to overcome some of the difficulties encountered with today's CAD systems.

The supercyclides, in particular, appear to possess good potential for shape modelling. Their properties include

- Low algebraic and parametric degree.

- Four-sided rather than triangular parametric patches.

- Blending capabilities (already demonstrated).

- (Possibly) sufficient geometric flexibility for free-form modelling.

- Distinctive properties providing relatively easy methods of geometric control.

- Trivially easy generation of planar faceted approximations for visualization purposes, using the fact that patch corners are coplanar.

- Exact solutions to the inverse parameterization problem.

Further research on these surfaces will investigate their use in a piecewise manner for the modelling of free-form surfaces. But first, present work on the properties of some special cases of the supercyclides needs to be completed. It is currently possible to obtain parametric representations of these surfaces from their algebraic forms, but further work is needed on the reverse process. This will enable the development of geometric algorithms for supercyclides that can make use of whichever is the most convenient surface formulation for a particular purpose. Much scope remains for research into algebraic methods for constructing $G^{2}$-continuous piecewisedefined surfaces, and for study of the potential uses of the Darboux cyclides for shape design.

\section{REFERENCES}

Allen, S., and Dutta, D. (1996). Supercyclides and blending, Computer Aided Geometric Design, Vol. 14, No. 7, pp. 637-651.

Bajaj, C.L. (1993). The emergence of algebraic curves and surfaces in geometric design. In Martin, R.R. (ed.): Directions in Geometric Computing, Information Geometers Ltd., Winchester, UK.

Bloomenthal, J. (ed.) (1997). An Introduction to Implicit Surfaces, Morgan Kaufmann, San Francisco, CA.

Blutel, E. (1890). Recherches sur les surfaces qui sont en même temps lieux de coniques et enveloppes de cônes du second degré, Ann Sci. Ecole Norm. Super., Vol. 3, No. 7, pp. 155-216.

Dahmen, W., and Thamm-Schaar, T.-M. (1993). Cubicoids: Modelling and visualization, Computer Aided Geometric Design, Vol. 10, No. 2, pp. 93-108. 
Darboux, G. (1896). Sur une Classe Remarquable de Courbes et de Surfaces Algébriques, $2^{\text {nd }}$ edition, Gauthier-Villars, Paris.

Degen, W.L.F. (1982). Surfaces with a conjugate net of conics in projective space, Tensor, N.S., Vol. 39, pp. 167-172.

Degen, W.L.F. (1986). Die zweifachen Blutelschen Kegelschnittflächen, Manuscripta Mathematica, Vol. 55, pp. 9-38.

Degen, W.L.F. (1994). Generalised cyclides for use in computer aided geometric design. In Bowyer, A.D. (ed.): Computer Aided Surface Geometry and Design, pp. 349-363, Oxford University Press (Proc. 4th IMA Conf. on the Mathematics of Surfaces, Bath, UK, Sept. 1990).

Degen, W.L.F. (1998). On the origin of supercyclides. In Cripps, R.J. (ed.): Mathematics of Surfaces VIII, Information Geometers Ltd., Winchester, UK (Proc. 8th IMA Conf. on the Mathematics of Surfaces, Birmingham, UK, Aug/Sept. 1998).

Dutta, D., Martin, R.R. and Pratt, M.J. (1993). Cyclides in surface and solid modeling, IEEE Computer Graphics \& Applications, Vol. 13, No.1, pp. 53-59.

Farin, G. (1993). Curves and Surfaces for Computer Aided Geometric Design, $3^{\text {rd }}$ edition, Academic Press.

Froumentin, M. and Chaillou, C. (1997). Quadric surfaces: A survey with new results. In Goodman, T.N.T. and Martin, R.R. (eds.): The Mathematics of Surfaces VII, Information Geometers Ltd., Winchester, UK (Proc. 7th IMA Conf. on the Mathematics of Surfaces, Dundee, Scotland, Sept 1996).

Guo, B. (1991). Modeling Arbitrary Smooth Objects with Algebraic Surfaces, $\mathrm{PhD}$ thesis, Dept. of Computer Science, Cornell University, Ithaca, NY.

ISO (1994). ISO 10303:1994 - Industrial Automation Systems and Integration - Product Data Representation and Exchange, International Organization for Standardization, Geneva, Switzerland. (The ISO catalogue is at http://www.iso.ch/cate/cat.html; search on 10303 for a listing of parts of the standard).

Kummer, E. (1864). Ueber die Flächen vierten Grades, auf welchen Schaaren von Kegelschnitten liegen, J. reine und angewandte Mathematik (Crelle/Borchardt), Vol. LXIV, pp. 66-76.

Malraison, P. (1998). A bibliography for $n$-sided surfaces. In Cripps, R.J. (ed.): The Mathematics of Surfaces VIII, pp. 419-430, Information Geometers Ltd., Winchester, UK (Proc. 8th IMA Conf. on the Mathematics of Surfaces, Birmingham, UK, Sept. 1998).

Martin, R.R., de Pont, J.J. and Sharrock, T.J. (1986). Cyclide surfaces in computer aided design. In Gregory, J.A. (ed.): The Mathematics of Surfaces, Oxford University Press (Proc. 1st IMA Conf. on the Mathematics of Surfaces, Manchester, UK, Sept. 1984).

Menon, J.P. (1994). Constructive shell representations for freeform surfaces and solids, IEEE Computer Graphics \& Applications, Vol. 14, No. 2, pp. 24-36.

Owen, J. (1997). STEP: An Introduction, $2^{\text {nd }}$ edition, Information Geometers Ltd., Winchester, UK.

Paluszny, M. and Boehm, W. (1998). General cyclides, Computer Aided Geometric Design, Vol. 15, No. 7, pp. 699-710.

Piegl, L. and Tiller, W. (1995). The NURBS Book, Springer-Verlag.

Pratt, M.J. (1990). Cyclides in computer aided geometric design, Computer Aided Geometric Design, Vol. 7, Nos. 1-4, pp. 221-242.

Pratt, M.J. (1995). Cyclides in computer aided geometric design II, Computer Aided Geometric Design, Vol. 12, No. 1, pp. 1-22.

Pratt, M.J. (1996). Dupin cyclides and supercyclides. In Mullineux, G. (ed.): The Mathematics of Surfaces VI, pp. 43-66, Oxford University Press (Proc. 6th IMA Conf. on the Mathematics of Surfaces, Brunel University, Uxbridge, UK, Sept. 1994). 
Pratt, M.J. (1997). Quartic supercyclides I: Basic theory, Computer Aided Geometric Design, Vol. 14, No. 7, pp. 671-692.

Pratt, M.J. (2001). Quartic supercyclides II: Special and degenerate cases. Manuscript, in preparation.

Pratt, M.J. and Geisow, A.D. (1986). Surface/surface intersection problems. In Gregory, J.A. (ed.): The Mathematics of Surfaces, Oxford University Press (Proc. 1st IMA Conf. on the Mathematics of Surfaces, Manchester, UK, Sept. 1984).

Rockwood, A.P. (1997). Blending. In Bloomenthal, J. (ed.): An Introduction to Implicit Surfaces, pp. 197-221, Morgan Kaufmann, San Francisco, CA.

Sederberg, T.W. (1985). Piecewise algebraic surface patches, Computer Aided Geometric Design, Vol. 2, Nos. 1-3, pp, 53-60.

Sederberg, T.W. (1990). Techniques for cubic algebraic surfaces: Parts 1 and 2, IEEE Computer Graphics \& Applications, Vol. 10, No. 5, pp. 14-25 and Vol. 10, No. 6, pp. 1221.

Sederberg, T.W. and Anderson, D.C. (1985). Steiner surface patches, IEEE Computer Graphics \& Applications, Vol. 5, No. 5, pp. 23-36.

Sederberg, T.W., Anderson, D.C. and Goldman, R.N. (1984). Implicit representation of parametric curves and surfaces, Computer Vision, Graphics \& Image Processing, Vol. 28, $72-84$.

Sederberg, T.W., Anderson, D.C. and Goldman, R.N. (1985). Implicitization, inversion and intersection of planar rational cubic curves, Computer Vision, Graphics \& Image Processing, Vol. 31, 89-102.

Shene, C.-K. (1997). Blending with affine and projective Dupin cyclides, Neural, Parallel and Scientific Computation, Vol. 5, Nos. 1-2, pp. 121-152.

Várady, T. (1983). Surface-surface intersections for double-quadratic parametric patches in a solid modeller. In Proc. $3^{\text {rd }}$ Anglo-Hungarian Seminar on Computer Aided Geometric Design, Cambridge, UK, Sept. 1983, Cambridge University Engineering Dept.

Warren, J. (1990). A Bound on the Implicit Degree of Polygonal Bézier Surfaces, Technical Report, Department of Computer Science, Rice University, Houston, TX.

Weatherburn, C.E. (1927). Differential Geometry of Three Dimensions, Vol. 1. Cambridge University Press. 